\title{
Effect of Ultrasound on Rough Rice Moisture Absorption Rate during Initial Soaking
}

\author{
A.H.M.N. Chamara ${ }^{1 *}$ and D.A.N. Dharmasena ${ }^{2}$
}

\begin{abstract}
Discharge of untreated wastewater to the environment, especially to the natural water bodies, is common in most of the small and medium scale parboiled rice processing facilities in Sri Lanka although the regulations do not permit such discharge. Effluents are added to water during soaking in the parboiling process where raw paddy is soaked in stagnant cold water for 36 to 72 hours or 2 to 4 hours in hot water at $60-65{ }^{\circ} \mathrm{C}$. Soaking followed by steaming and drying are the main steps of rice parboiling and this pregelatinization process reduces rice breakage during milling. Hot soaking can reduce water consumption by $91.6-95.8 \%$ and soaking time by $95 \%$. However, hot soaking needs significantly high energy and special equipment including a boiler and therefore, it is not popular among the small-scale millers. The small-scale millers are accountable for about $70 \%$ of rice milling in the country and about $55 \%$ of rice is consumed as parboiled rice. Due to high prevalence of cold-water soaking method, adverse environmental effects could occur as a result of discharging of waste water. Therefore, finding solutions to minimize the impact is essential. Recent studies have shown evidence that different types of grain soaking can be
\end{abstract}

Department of Biosystems Engineering, Faculty of Agriculture and Plantation Management, Wayamba University of Sri Lanka, Gonawila 60170, Sri Lanka ${ }^{2}$ Department of Agriculture Engineering, Postgraduate Institute of Agriculture, University of Peradeniya, Sri Lanka;

*nipuna@wyb.ac.lk improved by applying ultrasound waves during soaking. The objective of this research was to investigate the influence of moisture absorption rate of rough rice during cold soaking when combined with sonication to reduce soaking time, water usage and effluent generation. The laboratory studies revealed that ultrasound treatment could increase the soaking rate significantly. However, this is mainly due to the temperature rising during sonication and not due to the cavitation shear forces causing high sound pressure. When the temperature of water was maintained at room temperature, the soaking rate increased only slightly. Therefore, this study proved that sonication improved soaking rate of paddy.

Keywords: Parboiling, Rice, Sonication, Ultrasound

\section{INTRODUCTION}

Rice is the most widely consumed cereal grain in the world and in Sri Lanka. About 952.1 million tons of rice was produced in the year 2016 in the world, while Sri Lanka contributed from 4.2 million tons (FAO, 2016). Rice is consumed as raw rice or parboiled rice. There were no official statistics about the global consumption of parboiled rice, but in Sri Lanka, about $55 \%$ of rice is consumed in the parboiled form. Parboiling is the process of pre-gelatinizing the rice within the husk (Wimberly, 1983). 


\section{Effect of Ultrasound on Rough Rice Moisture Absorption Rate}

Raw rice is usually processed from paddy without any treatment but parboiled rice is processed by subjecting paddy to physicochemical treatment. Soaking, steaming and drying are the three main steps of rice parboiling process. Soaking is the process of enrichment of moisture content of the rice kernel above $30 \%$ to facilitate the gelatinization process before steaming.

There are two ways of soaking of paddy in Sri Lanka and the most widely practiced method used by the small to medium scale millers is cold soaking due to low investment. Paddy is kept in water at room temperature for 36 to 72 hours depending on the grain type and then, it is steamed for $4-5$ minutes before drying. Risk of fermentation, foul odour, and off flavour in the final product and high wastewater generation are the disadvantages of this normal cold soaking process. In the hot soaking process, the moisture content increases up to $30-35 \%$ within $2-4$ hours depending on the variety. It completely eliminates the foul odour but the cost of operation is considerably high compared to cold soaking. It is still necessary to recirculate water with reheating (Wimberly, 1983).

Roomi et al. (2007) suggested that parboiling is a high energy consuming process. Thermal energy consumption in the modern and semi-modern rice mills for parboiling varies in the ranges of 258.01 $\mathrm{MJ} /$ ton to $332.92 \mathrm{MJ} /$ ton and $312.19 \mathrm{MJ} / \mathrm{ton}$ to $446.19 \mathrm{MJ} / \mathrm{ton}$, respectively. Similarly, thermal energy consumption of the modern and semi modern rice mills for drying varies from 519.40 MJ/ton to $703.89 \mathrm{MJ} /$ ton and $694.12 \mathrm{MJ} /$ ton to $750.60 \mathrm{MJ} /$ ton respectively (Roomi et al., 2007). Steaming and drying accounts for more than $3 / 4^{\text {th }}$ of total energy requirement. Based on the literature, it is obvious that the steaming time can be reduced if the moisture content after the soaking step is high. At the same time, by reducing the soaking time, effluent discharge can be reduced.

Various energy efficient effluent elimination techniques are available in the literature. Sonication is a technology which is being used in the food industry for different purposes such as wastewater purification, extraction of anthocyanin and antioxidants, speed dissolution, and dispersion to save expensive emulgators (e.g. mayonnaise) or to speed up filtration processes (e.g. vegetable oil etc.). Sonication is the process of applying ultrasound waves to an object or a solution and the literature revealed that the effects of sonication on grain soaking need to be analysed to properly understand the relationships between different parameters and phenomenon behind it.

It was found that sonication during cooking effectively reduces the cooking time of brown rice while maintaining high cooking quality by enhancing water diffusion to the rice kernel (Yu et al., 2015). Sonication can generate fluid mixing and generate shear forces through several effects. When rice grains are subjected to sonication, surface 
shells of the rice grain get destructed to some extent and grain fragmentation happens, causing a fast release of starch during cooking and it is the reason to have a less cooking time according to Yu et al. (2015). It is found that when large particles suspend in a solution which is sonicated, their surfaces are eroded (Mason et al., 1996). These erosions that may occur on paddy husk surface during the soaking process can increase hydration during soaking and steaming while reducing the time of drying due to the increase of diffusion rate through the paddy husk.

Ultrasound is physically a sound wave which has frequencies above $20 \mathrm{KHz}$ and has different amplitudes based on the energy carry in the wave. Commonly, ultrasound is generated by applying an electrical energy to a piezoelectric material called as transducer. Then, the piezoelectric material converts the electrical energy supplied to it to mechanical vibration and it moves the air layers adjacent to it in the same frequency. The transducer is bonded to a surface that can transmit this vibration through the fluid. Due to the power transmitted as the mechanical vibration energy, the liquid subjected to ultrasound treatment gets heated unless it is cooled by a separate mechanism.

In the laboratory, two types of ultrasonic devices are generally employed. In an ultrasonic horn, a single transducer is placed within a titanium cylinder, which results in an intense acoustic field in the region immediately below the device, with a vibration amplitude of approximately $100 \mu \mathrm{m}$ (Hunter et al., 2008). An ultrasonic horn can produce a very high energy intensity directly below the device, but this intensity decay significantly with the distance from the source. Ultrasonic bath (Sonication Tub, Figure 1) delivers more diffuse acoustic energy through a distribution of several transducers spread across the base of the bath. These systems generally provide much lower energy intensity.

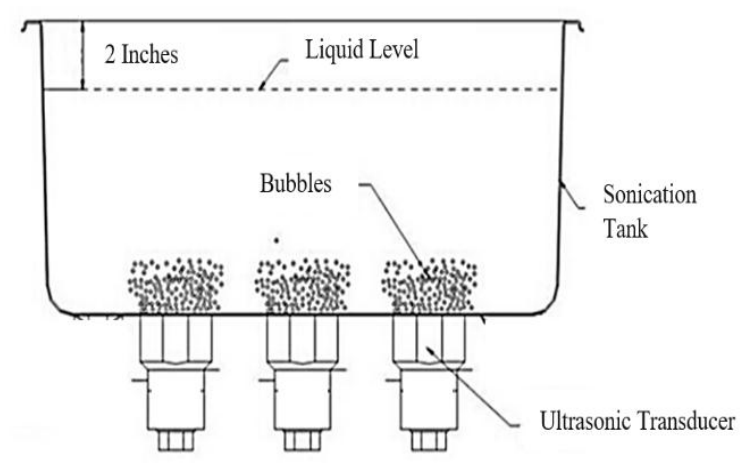

Figure 1. Sonication tub

Ultrasound (US) can generate fluid mixing and shear forces through several effects. Primarily, a sound wave is a wave of low- and high-pressure regions moving through a liquid. The rapid changes in pressure cause fluid motion referred to as acoustic streaming. However, cavitation is usually of greater importance in food processing applications. This is the formation of small bubbles at the points of low pressure in the sound wave.

Kentish and Feng (2014) suggest that these bubbles form because the liquid 
medium is incompressible and cannot readily accommodate the rapid changes in system pressure. The sizes of cavitation bubbles are determined by the ultrasound frequency, and a cavitation liquid can contain many thousands of such bubbles (Kentish and Feng, 2014). This experiment was designed to study the effect of ultrasound on soaking rate of paddy. The main hypothesis set was that the ultrasound can enhance the soaking rate of paddy.

\section{MATERIALS AND METHODS Sample Preparation}

BG-300 rice variety with $12 \pm 0.5 \%$ dry basis moisture content was obtained from a farmer's store, Anamaduwa, North Western Province, Sri Lanka.

Selected rice was cleaned manually. Only the kernels of BG 300 rice were separated and stored in room temperature in plastic containers until they were used for study. Prior to the use for all the soaking processes, the kernels were thoroughly washed by tap water to remove dust particles attached to the husk of the grains.

\section{Ultrasound Treated Soaking of Rough Rice Kernels}

$200 \pm 10 \mathrm{~g}$ of cleaned rice was added to the soaking bath (Figure 1) and water at room temperature was added while keeping the water level 2 inches above the bottom of the soaking tub. MRC DCG-120H Ultrasound bath was used to soak paddy to expose rice grains to US waves in Auto-Degas Mode (ULTRASONIC-CLEANERS Digital, Degas function. Available at: http://mrclab.com/data /products/DCG-80H_SPEC.pdf (verified 21 Jun. 2017)). Temperature was recorded throughout the whole soaking process continuously. The frequency of US wave used was $40 \mathrm{kHz}$.

\section{Soaking of Rough Rice Kernels}

As shown in figure 2, $200 \pm 10 \mathrm{~g}$ of cleaned paddy was added to the soaking bath and water at room temperature was added while keeping the water level 2 inches below the top of the soaking tub. Cold soaking tub had the same dimensions of the sonicater soaking tub. Temperature was recorded during the whole soaking process continuously. This was the control experiment to compare the results of ultrasound exposed soaked paddy.

\section{Temperature Measurement and Recording}

Waterproof DS18B20 sensors with a programmed Arduino mega 2560 module was used to transmit temperature readings to the computer for logging.

\section{Temperature Effect Removed Soaking of Rough Rice Kernels}

Initial soaking readings were taken without controlling the temperature. In order to remove the effect of temperature, continuous water replacement was performed on both 
cold soaked process and US assisted soaking (Figure 2).

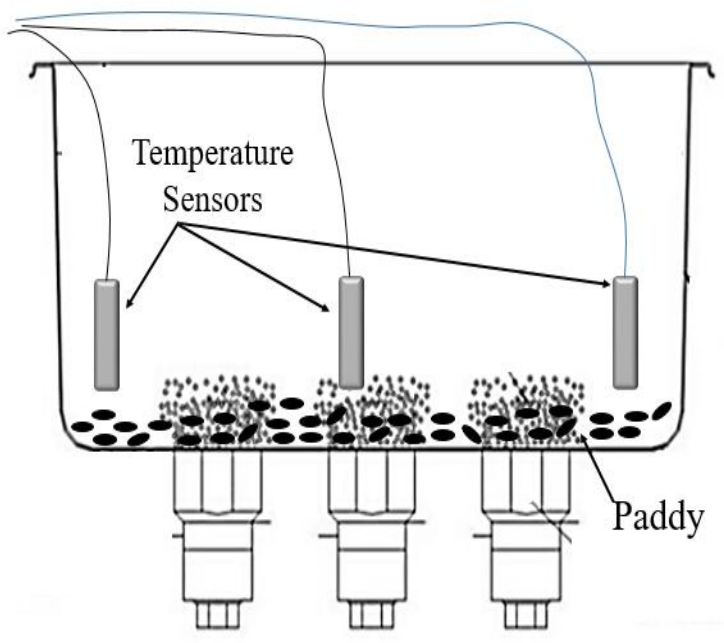

Figure 2. Normal cold soaking

Flexible hoses of 0.5 -inch diameter were used for water inlet and outlet. Ultimately, there were four soaking processes. They are Normal Cold Soaking (NCS), Normal Ultrasound Assisted Soaking (NUSAS), Temperature Controlled Cold soaking (TCCS), and Temperature Controlled Ultrasound Assisted Soaking (TCUSAS) (Figure 3).

\section{Measurement of Moisture Content of Rice Kernels}

Paddy samples were obtained from controlled and experimental tests, cold soaking vs US exposed soaking within equal time periods after the experiment was started. Water was drained from the sample and it was pressed between paper towels to remove surface moisture in the outer husk. Then, the sample was ground using a mortar and pestle before testing the moisture in a halogen light moisture meter. Wet-basis percentage of moisture was measured and recorded with the time of sample taken.

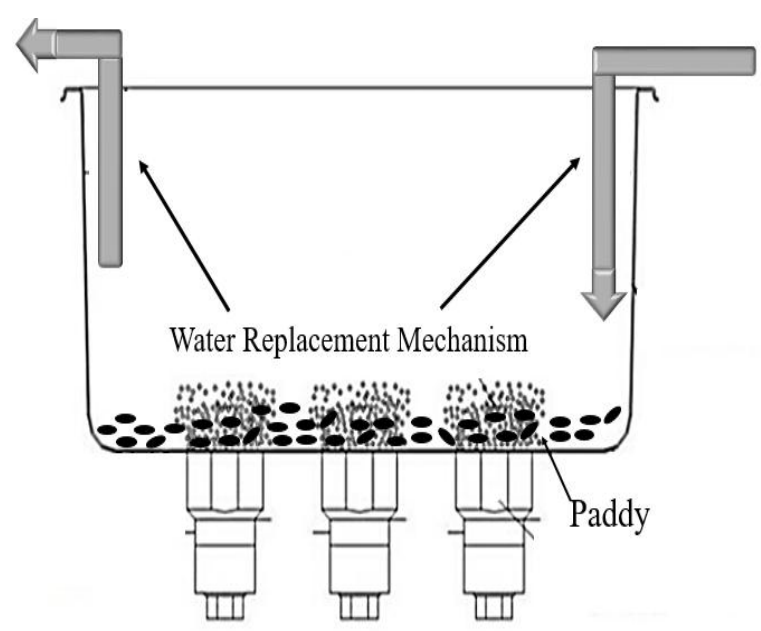

Figure 3. Temperature controlling by continuous water replacement

\section{RESULTS AND DISCUSSION}

Two data sets were obtained during this study. Figure 4, shows the moisture percentages in cold soaked process and US assisted soaking without temperature control. Figure 5 represents moisture percentage with temperature control.

\section{Temperature Change during Soaking}

During the experiments, the room temperature varied between $28-32{ }^{\circ} \mathrm{C}$. However, the temperature of water remained 
as $28 \pm 1{ }^{\circ} \mathrm{C}$ during the experiments. In normal cold soaking procedure, the soaking solution temperature increased by $6^{\circ} \mathrm{C}$. It is above 2 ${ }^{\circ} \mathrm{C}$ compared to the maximum room temperature while the rate of temperature change was about $0.5^{\circ} \mathrm{C} / \mathrm{min}$. In the NUSAS process, the temperature reached to a maximum of $72{ }^{\circ} \mathrm{C}$. During the temperature controlled soaking processes, continuously supplied water was able to maintain the TCCS soaking solution temperature at $28 \pm 1$ ${ }^{\circ} \mathrm{C}$ while the TCUSAS soaking solution was maintained at $30 \pm 2{ }^{\circ} \mathrm{C}$. The water replacement arrangement affected the soaking rate in a negligible rate as the water flow velocity near soaking paddy was kept near $0 \mathrm{~m} / \mathrm{s}$.

Table 1. Rate of moisture increase with the soaking method

\begin{tabular}{lcc}
\hline $\begin{array}{l}\text { Soaking } \\
\text { Method }\end{array}$ & $\begin{array}{c}\text { Rate of moisture } \\
\text { increase }(\% / \\
\text { minute) }\end{array}$ & Notation \\
\hline NUSAS & 0.0405 & $\mathrm{M}_{1}$ \\
NCS & 0.0319 & $\mathrm{M}_{2}$ \\
TCUSAS & 0.0356 & $\mathrm{M}_{3}$ \\
TCCS & 0.0233 & $\mathrm{M}_{4}$ \\
\hline
\end{tabular}

Normal Ultrasound Assisted Soaking (NUSAS), Normal Cold Soaking (NCS), Temperature Controlled Cold soaking (TCCS), and Temperature Controlled Ultrasound Assisted Soaking (TCUSAS)

\section{Moisture Content during Soaking}

Figure 4 represent the relationship between temperature uncontrolled cold soaking and ultrasound assisted soaking moisture increment. Figure 5 represent the temperature controlled or continuously water passed cold soaking and ultrasound assisted soaking process moisture increment.

$$
\begin{array}{lll}
\mathrm{Y}=0.0356 X+15.789 & \mathrm{R}^{2}=0.9059 & \text { TCUSAS } \\
\mathrm{Y}=0.0319 X+15.505 & \mathrm{R}^{2}=0.9479 & \text { NCS } \\
\mathrm{Y}=0.0405 X+15.258 & \mathrm{R}^{2}=0.9280 & \text { NUSAS } \\
\mathrm{Y}=0.0233 X+15.789 & \mathrm{R}^{2}=0.9059 & \text { TCCS }
\end{array}
$$

According to the derived equations, the rate of moisture absorption is in the $\mathrm{M}_{4}<\mathrm{M}_{2}$ $<\mathrm{M}_{3}<\mathrm{M}_{1}$ order.

In both, temperature controlled, and uncontrolled ultrasound assisted soaking methods (NUSAS \& TCUSAS) have higher moisture absorption rates than cold soaking processes (NCS and TCCS). It is an evidence that US soaking can improve the soaking rate of rice. The temperature effect during ultrasound assisted soaking can increase the soaking rate because $M_{1}$ is greater than $M_{3}$.

Wambura, et al. (2008), proved that there is a clear improvement of soaking rate of different grains without husks due to sonication. According to this experiment it is positive that similar approach can be used to rough rice to improve the soaking rate.

\section{Limitations}

MRC DCG-120H ultrasound bath can operate only 120 minutes continuously or up to the maximum manufacturer allowable temperature continuously. It limited the rice soaking time to maximum 120 minutes in this experiment. 


\section{A.H.M.N. Chamara and D.A.N. Dharmasena}

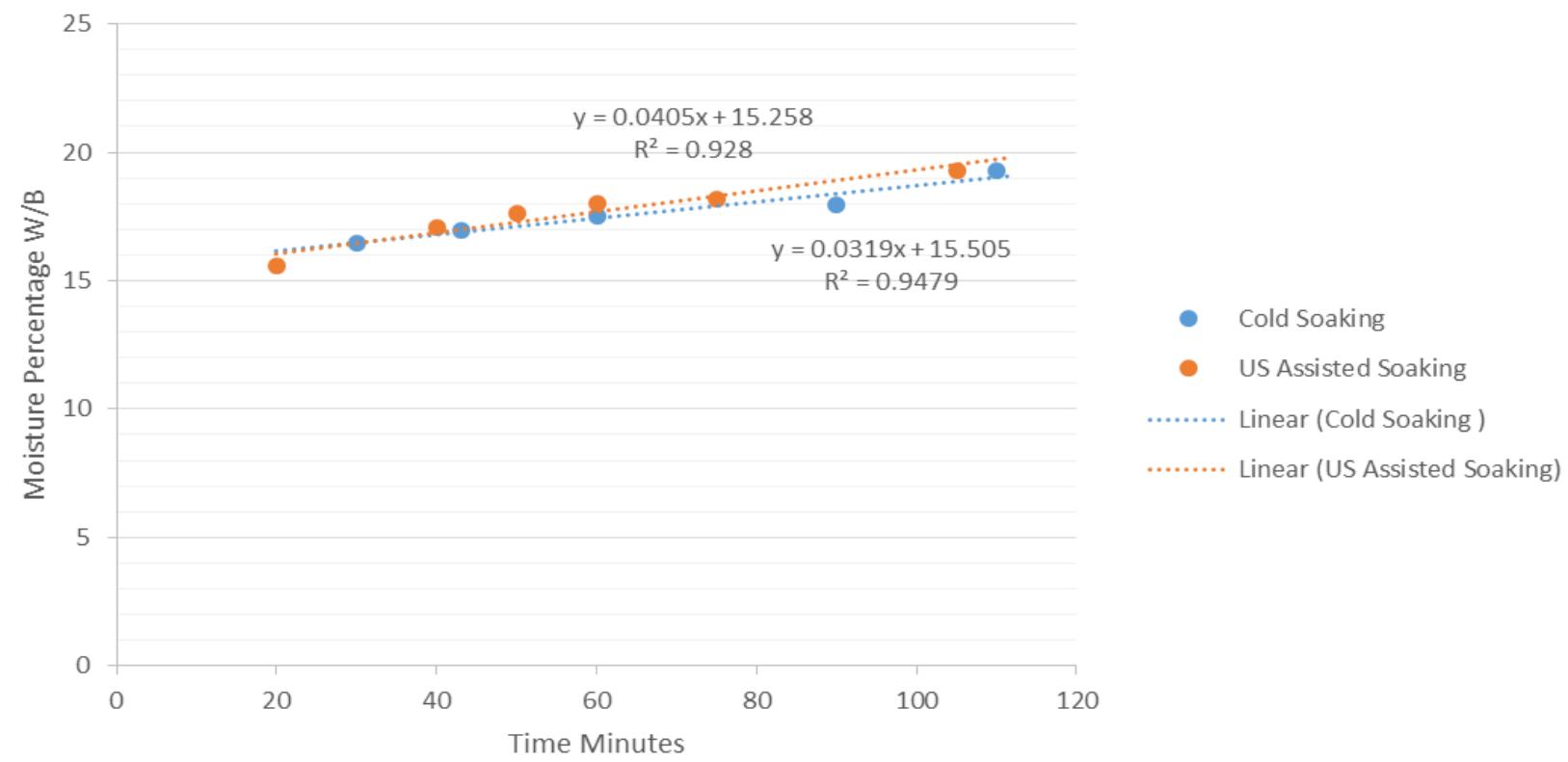

Figure 4. NCS and NUSAS moisture percentage with time

NCS - Normal Cold Soaking (M2), NUSAS - Normal Ultrasound Assisted Soaking (M $M_{1}$

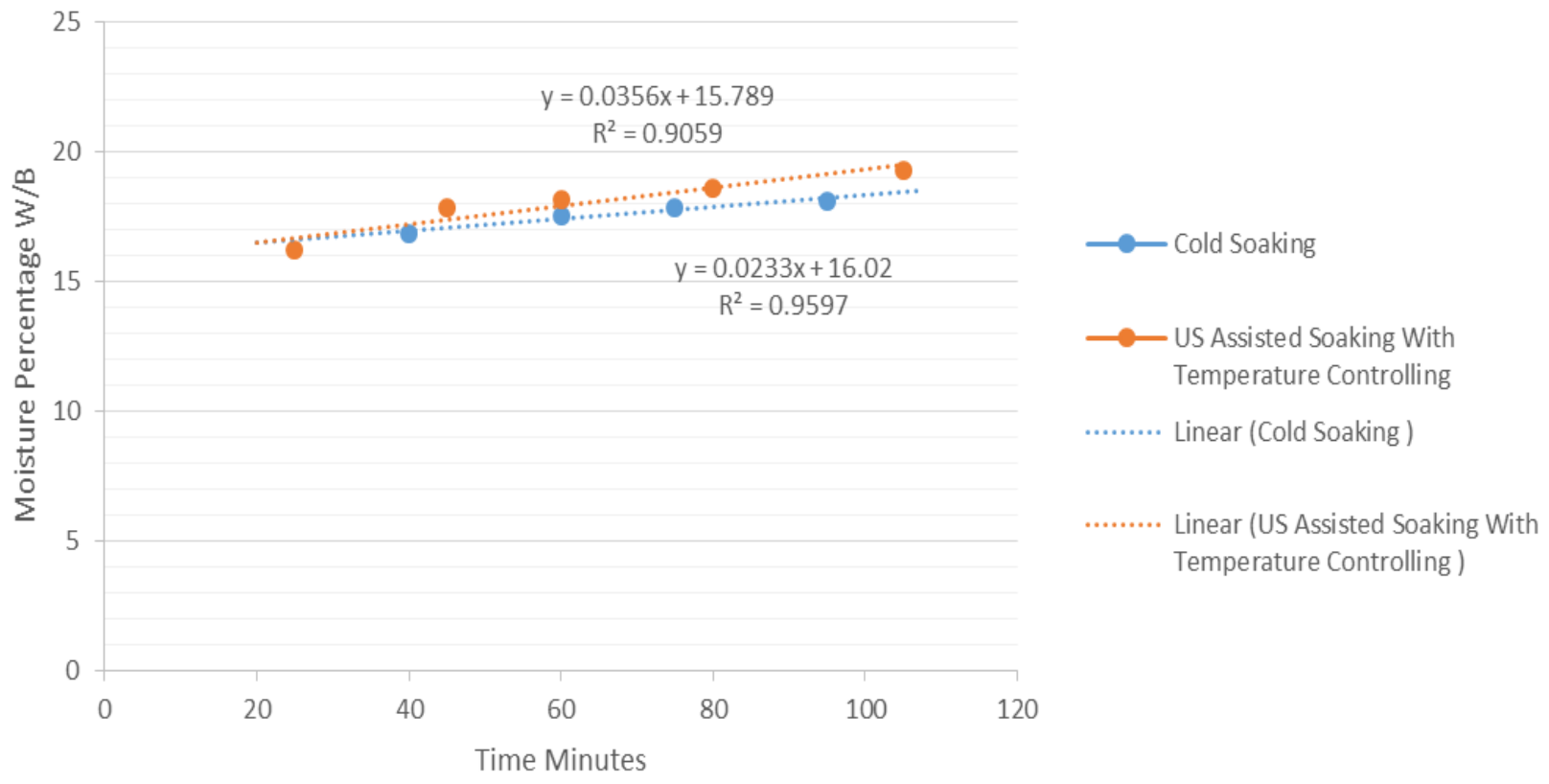

Figure 5. TCCS and TCUSAS moisture percentage with time

TCCS - Temperature Controlled Cold soaking (M4), TCUSAS - Temperature Controlled Ultrasound Assisted Soaking (M3) 


\section{Effect of Ultrasound on Rough Rice Moisture Absorption Rate}

\section{CONCLUSION}

The results proved that the sonication can increase the soaking rate of paddy. Removal of foreign matter attached to paddy husk, releasing of air bubbles attached to paddy husk may be the reason to the increment of the moisture absorption rate.

During the initial soaking stage, the effect of ultrasound alone did not significantly increase the soaking rate. Temperature of water also played a critical role to increase the soaking rate. Authors suggest that the effects of ultrasound like shearing, bubble forming, and erosions convert acoustic energy to heat at the end. Therefore, the whole effect of ultrasound for soaking can be used for reducing the soaking time of paddy.

According to the experiment although initial soaking rates of temperature controlled cold soaking is higher than temperature controlled cold soaking if the soaking time increase further there will be a decline trend in normal cold soaking moisture absorption due to effluent stagnation.

This experiment revealed that there is a potential to apply sonication to improve the paddy soaking process but it is too early to say that sonication is commercially and technologically viable in real industry. Considering the limitations, further studies are needed in order to study the effect of sonication for overall soaking time period.
Also, the soaking rate needs to be studied under horn type ultrasound transducer as well. Further, soaking rate needs to be studied under different frequencies to identify the best wave form.

\section{REFERENCES}

Food and Agriculture Organization of the United Nations. (2016) Available at: http://www.fao.org/faostat/en/\#data/QC [Accessed $1^{\text {st }}$ March 2017].

Hunter, G., Lucas, M., Watson, I. and Parton, R. (2008). A radial mode ultrasonic horn for the inactivation of Escherichia coli K12. Ultrasonics Sonochemistry, 15(2): 101-109.

Kentish, S. and Feng, H. (2014). Applications of power ultrasound in food processing. Annual Review of Food Science and Technology, 5: 263-284.

Mason, T.J., Paniwnyk, L. and Lorimer, J.P., (1996). The uses of ultrasound in food technology. Ultrasonic Sonochemistry, 3: 253-260.

Roomi, M.S.M., Namal, D.D.A. and Jayasinghe, K.T. (2007). Study of energy consumption pattern in Sri Lankan rice mills - Enhancing opportunity for conservation. Engineer, 40(1): 83-88.

ULTRASONIC-CLEANERS Digital, Degas function. Available at: http://mrclab.com/data/products/DCG80H_SPEC.pdf (verified 21 Jun. 2017). Wambura, P., Yang, W. and Wang, Y., (2008) Power ultrasound enhanced onestep soaking and gelatinization for rough 
rice parboiling. International Journal of Food Engineering, 4(4): DOI: https://doi.org/10.2202/1556-

3758.1393.

Wimberly, J., (1983) Technical hand book for the paddy rice post-harvest industry in developed countries. 1st ed. Manila: International rice research institute.

Yu, Y., Ge, L., Zhu, S., Zhan, Y. and Zhang, Q. (2015). Effect of presoaking high hydrostatic pressure on the cooking properties of brown rice. Journal of Food Science and Technology, 52(12): 7904-7913. 